\title{
THERMOSENSITIVE HYDROGELS-A POTENTIAL CARRIER FOR THE DELIVERY OF DRUGS AND MACROMOLECULES
}

\author{
SOUMYA RANJANA SAHOO, MOTHILAL M. ', PRIYADHARSHINI B., DAMODHARAN N.
}

Department of Pharmaceutics, SRM College of Pharmacy, SRM Institute of Science and Technology, Kattankulathur, 603203, Tamilnadu, India *Email: mothipharma78@gmail.com

Received: 03 Nov 2020, Revised and Accepted: 13 Feb 2021

\begin{abstract}
In this review, the authors have discussed scientific advances in thermosensitive hydrogels over the past two decades. The ability of the thermosensitive hydrogel to undergo rapid changes with response to temperature makes it an attractive candidate for many biomedical applications such as targeted drug delivery, wound healing, soft contact lenses, sensors, tissue regeneration, gene, and protein delivery. This review aims to deliver a brief overview of gelation properties, merits, and demerits of various natural and synthetic thermo-sensitive polymers that have significant clinical relevance. The report emphasizes the importance of injectable thermosensitive hydrogels, as it can offer improved solubility of hydrophobic drugs and site-specificity, extended-release of drugs and macromolecules, improved safety, and local administration of drugs. The authors has also provided a commentary on the delivery of drugs or macromolecules from thermo-sensitive hydrogels through various approaches. This review highlights the current status of research in thermo-sensitive hydrogels and emphasizes the importance of developing nontoxic thermo-sensitive hydrogels, dual responsive, and multi-responsive hydrogel systems.
\end{abstract}

Keywords: Thermo-sensitive hydrogels, Gelation property, Delivery of drugs, Tissue regeneration

(C) 2021 The Authors. Published by Innovare Academic Sciences Pvt Ltd. This is an open access article under the CC BY license (https://creativecommons.org/licenses/by/4.0/) DOI: https://dx.doi.org/10.22159/ijap.2021v13i2.40162. Journal homepage: https://innovareacademics.in/journals/index.php/ijap

\section{INTRODUCTION}

Hydrogels are water-insoluble, randomly cross-linked threedimensional polymeric systems that have an incredible capability to swell and retain a significant amount of water within their structural framework. The water molecules entrapped into the hydrogel network crosslinks the macromolecules through physical or chemical means [1, 2]. The basic properties of hydrogels are can be brought under mechanical properties, biocompatibility properties, and swelling properties [3]. Hydrogels containing nanoparticles should be used to optimize the thermal, mechanical, optical, and chemical properties of the hydrogel [4]. Physical cross-linking of hydrogels results from weak interactions such as hydrogen bonds, hydrophilic/hydrophobic interactions, ionic/electrostatic interactions, reversible intermolecular interactions, stereo-complex formation, metal coordination, $\pi-\pi$ stacking, and polymerized entanglements. The physically cross-linked hydrogels can be easily disrupted by adding organic solvents or by changing the ionic strength, $\mathrm{pH}$, or temperature owing to their weak cross-linking interaction. Chemical crosslinking results in the formation of stable hydrogel with considerable mechanical strength and are usually created by photo-polymerization; Diels-Alder clicks reaction, Michael type addition, oxime formation, Schiff base formation, and enzyme-induced crosslinks [2, 5]. Placing hydrophilic groups such as amides, sulfonic acids, hydroxyl groups, and carboxylic acids into the structure, hydrogels can absorb large amounts of water and form hydrophilic polymers [6]. It can be added through novel mechanisms that can be used to improve the physical properties of the hydrogel [7]. Hydrogels are biocompatible and biodegradable, resembling natural tissues due to their high flexibility and high moisture content. Hydrogels offer good transport properties, making them a potential drug delivery carrier to achieve timed release of drugs or nutrients [8]. Hydrogel has gained a special interest among the biomedical community due to their extensive property and their resemblance with the extracellular matrix [9]. Useful applications of hydrogels include drug delivery devices and wound dressings [10,11]. Some of the limitations associated with hydrogels are lower mechanical strength, difficulty to handle, and difficult to load with nutrients or drugs. To overcome these limitations, smart hydrogels are developed [12].

In this review, the authors have delved into the scientific developments of thermosensitive hydrogels during the past two decades. The major focus was the newer thermosensitive hydrogels and advances in their applications as a tool in targeted and prolonged-release drug delivery. Thermo-sensitive hydrogels have received considerable attention due to their ease of application, simplicity of drug formulation, less antagonistic effect on tissues, protective environment for drugs, localized drug delivery, and other attractive gelling systems [13]. Depending on thermo-sensitive groups present, the formation of thermo-sensitive hydrogels momentarily happens by a microscopic mechanism when the gelation temperature is attained. The solid phase is separated from the solution near the critical temperature [14]. Thermo-sensitive hydrogels can be classified into negatively thermo-sensitive and positively thermo-sensitive hydrogels. Negatively thermo-sensitive hydrogels are formed by LCST polymers, whereas positively thermosensitive hydrogels are formed by UCST polymers [15]. At lower consolute temperature or lower critical solution temperature (LCST), the polymer becomes hydrophobic and shrinks due to change in the phase from soluble random coil to insoluble globule structure. The inherent LCST can be modified by changing the hydrophilic/hydrophobic ratio. In contrast, upper consolute temperature or upper critical solution temperature (UCST) polymers are enthalpy driven, which swells with heating and turns soluble in an aqueous system due to dissociation of interpolymer complex [16]. The sol-gel properties of thermosensitive hydrogels are non-invasive because they can liquefy the hydrogel almost anywhere in the CNS. Viscous hydrogels form a sustained-release reservoir of the therapeutic agent at body temperature. It can also be filled with a variety of drugs, including neuroprotective agents, neurotrophic factors, chemotherapeutic agents, and cells [17]. In certain physiological conditions, the in-situ (gel) delivery system involves the change of liquid state of the formulation into a gel at the site of application. Many factors regulate the gelation in the field, viz., temperature, $\mathrm{pH}$, solvent exchange, ion crosslinking, and UV light [18]. The sol-gel transition of hydrogels is determined using various testing strategies, including cloud and pour point estimation, rheological properties, and differential scanning calorimeter (DSC) [19-22]. In-situ delivery systems can significantly reduce the concentration and frequency of the daily dose, patient compliance, improving bioavailability and cost-effectiveness [23].

The characteristic phase transition of polymers enables the development of smart medicated formulations such as splash, injectable, and shaped hydrogels which retain the drug at the site of utilization over a longer period [24]. The thermosensitive drug 
delivery system depends on the temperature change to release its payload through dissolution, diffusion, disintegration, and erosion mechanism [25]. An ideal thermosensitive hydrogel drug delivery system should stream freely at ambient temperature and transform into a non-streaming gel at physiological temperature $\left(32{ }^{\circ} \mathrm{C}-37{ }^{\circ} \mathrm{C}\right)$ [24]. This demands the development of in-situ hydrogel, which can readily arrange and harden inside the body with negligible protrusion [26]. The injectable thermosensitive hydrogel has gained special interest as it can offer improved solubility of hydrophobic medications, site-specificity, sustained-release behavior, delivery of drugs and macromolecules including protein, peptide, nucleic acid improved safety, etc [24], local administration to avoid the first-pass metabolism and easily administered without surgical procedure [27]. Some of the limitations associated with the system include increased surgical risk associated with device implantation/retrieval and chances of clogging inside the body immediately after injection [28].

The purpose of this review is to provide an overview of thermosensitive hydrogels, important thermosensitive materials, drug delivery approaches, and applications of thermosensitive hydrogels.

\section{Thermosensitive polymers}

Thermosensitive or thermo-responsive polymers undergo macroscopic changes in the aqueous medium when lower critical solution temperature or upper critical solution temperature is reached. The thermosensitive polymers are mainly categorized into natural and synthetic polymers.

\section{Natural polymers}

Natural polymers were widely studied because of their non-toxicity, biocompatibility, biodegradability, and low inflammatory response similar to that of host tissue. However, these polymers present immunological concerns, batch to batch variation, and difficulty in purification. The natural polymers can be easily modified to obtain a wide variety of applications. Interestingly, numerous researchers obtain hydrogels by combining natural polymers with synthetic polymers. The most commonly used natural thermo-responsive polymers are categorized into polysaccharides (Cellulose derivative, Chitosan, Dextran, Xyloglucan) and proteins (gelatin, collagen, and albumin) [29].

\section{a. Cellulose and cellulose derivative}

Cellulose is a naturally occurring water-insoluble polymer that has a greater degree of hydrophilicity on its chain structure. The insolubility of this highly hydrophilic cellulose is mainly due to the formation of strong intermolecular hydrogen bonds. The aqueous solubility of cellulose can be achieved by substitution of a certain fraction of hydroxyl groups by hydrophobic groups such as hydroxyl propyl groups or methyl groups, which in turn disrupts the intermolecular hydrogen bonds. Complete substitution of hydroxyl groups with hydrophobic moieties renders the cellulose insoluble [30]. Depending on the degree of substitution, the thermoreversibility of methylcellulose can take place in the temperature range from $60^{\circ}$ to $80^{\circ} \mathrm{C}$ [31]. The LCST of methylcellulose hydrogels can be adjusted to physiological temperature by grafting it with other monomers. Liu et al., grafted methylcellulose with $\mathrm{N}$-isopropyl acrylamide (NIPAM) in various ratios to adjust the LCST to the desired temperature [32]. The PNIPAM grafted with lower levels of methylcellulose decreased the LCST, whereas at higher levels the LCST increases. The inclusion of methylcellulose into the PNIPAM structure enhanced the mechanical stability of the gel without expulsion of the liquid. The higher LCST of cellulose derivatives limits their usage as thermo-sensitive gels. However, they are utilized as controllers to tune the other thermo-sensitive polymeric system to attain the desired LCST.

\section{b. Chitosan}

Chitosan, also known as Poliglusamis a natural poly-cationic linear oligosaccharide derived from chitin by alkaline hydrolysis. They are composed of randomly distributed acetylated and deacetylated Dglucosamine units bound with each other through $1 \rightarrow 4$ glycosidic linkages. The chitosan polymeric chains have many amine groups ($\left.\mathrm{NH}_{2}\right)$ and hydroxyl groups $(-\mathrm{OH})[33,34]$. The amine functional group of chitosan is highly reactive and allows the derivatization of polymers for improved properties such as bio-adhesion, mucoadhesion, gene transferability, high drug loading, and controlled drug release $[29,34]$. The biodegradability, biocompatibility, and low toxic potential of chitosan, make it preferable over other natural polymers [35, 33] despite their limitations such as small specific surface area and void fraction [36].

Chenite et al. (2000) were the first to explore the thermo-sensitive behavior of chitosan modified with glycerophosphate [37]. The gelation temperature remains unchanged with a varying molecular weight of chitosan, whereas it is greatly influenced by the concentration of glycerophosphate when the concentration and level of deacetylation of chitosan remain constant. The gelation temperature of the chitosan can be reduced with increasing concentration of the glycerophosphate $[38,39]$. Following Chenite et al. significant advancement, several researchers were involved in the development of thermo-sensitive hydrogels on natural polymers. The mechanism of gelation involved in thermo-responsive chitosan/polyol-phosphate system has been demonstrated by Nicolas Anton $e t$ al. They stated that a hydration defensive layer of polyols is held around the chitosan through weak intermolecular hydrogen bonding; any increase in temperature disrupts the hydrogen bonding of polymer and induces gelation through stronger hydrophobic interactions [40, 41]. Bhattarai et al. produced a thermoreversible hydrogel using polyethylene glycol and chitosan without the addition of a crosslinking agent [41]. Nazar et al. synthesized N-trimethyl chitosan chloride/polyethylene glycol/glycerophosphate hydrogels for nasal drug delivery, showing a thermoreversible behavior at $35{ }^{\circ} \mathrm{C}$ [42]. Though chitosan has lower mechanical strength, it is broadly utilized in the development of thermo-sensitive hydrogel systems.

\section{c. Xyloglucan}

Xyloglucan is non-ionic hemicellulose and a hydrophilic polysaccharide that carries xylose and galactosyl-xylose. They are abundantly found in primary cell walls and seeds of all vascular plants. A "mucin-like" xyloglucans obtained from tamarind seeds are linear $\beta$ - $(1 \rightarrow 4)$-D-glucan chain branched with (1-6)- $\alpha$-xylose or $(1-$ $2)-\beta$-galactoxylose side chains [43-45]. Xyloglucan is digested using fungal $\beta$-galactosidase to eliminate approximately $35 \%$ of the galactose residues [46, 47]. The transition temperature of xyloglucans is inversely proportional to the concentration of polymer and the percentage of galactose residues removed [48, 49]. Xyloglycans undergo a phase transition at a much lower concentration (1 to $3 \% \mathrm{wt}$ ) compared to other thermo-sensitive polymers, including block polymers [50,51]. Xyloglucans are highly biocompatible, biodegradable, and have high water absorption/retention capacity. It has drawn a considerable research interest in developing xyloglucan hydrogels for biomedical applications [52-55]. Additionally, xyloglucan hydrogels combined with poly D-lysine showing a phase transition under physiological conditions were assessed for their possibility to deliver the cells. Poly-D-lysine-incorporated xyloglucan hydrogels promote the repair of damaged neural pathways, including the axons in the central nervous system [56]. Derivatized xyloglucans are interconnected three-dimensional microporous systems that adhere to cells and detach when the suitable temperature is reached. The microporous structure of xyloglucans directs them to be a potential delivery vehicle in the use of regenerative medication $[51,57]$.

\section{d. Dextran}

Dextran is a biocompatible and biodegradable polysaccharide obtained from the enzymatic decomposition of sugarcane. Dextran lacks thermosensitive behavior; however, their incorporation into other thermosensitive materials form thermosensitive hydrogels [58]. Huang et al. prepared block polymers consisting of dextran, NIPAAm, 2-hydroxyethyl methacrylate (HEMA), oligolactate that are capable of forming thermosensitive hydrogels with LCST around $32{ }^{\circ} \mathrm{C}$. Wang et al. demonstrated the protective effects of block polymer comprising dextran, Polycaprolactone, and NIPAAm or HEMA on the remodeling of ventricular damage caused by myocardial infarction [59].

\section{e. Gelatin}

Gelatin, a thermo-reversible and cold setting polymer composed of a mixture of peptides and denatured proteins acquired by incomplete hydrolysis of collagen extracted from bones and connective tissues of animals [60]. Gelatin appears to be semi-solid beneath UCST $\left(35^{\circ} \mathrm{C}\right)$, 
as the gelatin molecules are inter and intramolecularly cross-linked by hydrogen bonds, forming a three-dimensional triple helix steady collagen supercoil structure. The cross-linking density of the gelatin is dependent on the number of water molecules entrapped and bound to $\mathrm{NH}_{2}$ groups of supercoiled chains via hydrogen bonding [61, 62] Gelatin undergoes conformational changes from a triple helix to a random coil at $40^{\circ} \mathrm{C}$, introducing a fluidic state [61]. The fluidic state of gelatin offers a large number of reactive functional groups, allowing them for modification of gelation properties [27]. Yang and Kao developed a hydrogel composed of poly (ethylene glycol)-poly (D, Llactide) block copolymer and gelatin, which flows effortlessly at $37^{\circ} \mathrm{C}$ and remains gel at room temperature [63].

\section{Synthetic polymers}

Synthetic thermo-sensitive polymers are biodegradable and have a considerable mechanical strength depending on the molecular weight, concentration, and ratio of molecular units. Though the LCST of these polymers can be easily modified to the desired temperature, most of these polymers are non-biocompatible and requires the incorporation of natural polymers for their use as thermosensitive injectable hydrogels [59].

The various thermosensitive synthetic polymers include NIPAMbased system, PEO-PPO based system, PEG-biodegradable polyester, dimethylamino based system, poly (organophosphazene), pluronic hydrogel, PEG-polyester, Polyacrylamide derivative, 2-hydroxy ethyl and methyl methacrylate, trimethyl-ol-propane, trimethacrylate, benzoin methyl ether, polyurethane, polyvinyl pyrrolidone, PLGA, Poly (N, N-diethyl acrylamide), Poly (N-ethyl methacrylamide), Poly (methyl vinyl ether), poly (2-ethoxy ethyl vinyl ether), Poly (N-vinyliso-butyramide), Poly (N-vinyl caprolactam), Poly (N-(2-hydroxy propyl) methacrylamide mono/dilactate), Poly (acrylic acid-coacrylamide), poly-ethers, Poly (alkyl oxide) copolymers, C Pluronic F-127, Poly (Dimethylamino ethyl methacrylate) [64-68]. Thermoresponsive synthetic polymers are generally amphiphilic triblocks of poly (ethylene oxide) (PEO), poly (propylene oxide) (PPO), poly-lactic acid (PLA), Poly (D, L-lactic acid (CO-glycolic acid)) (PLGA), poly (e-caprolactone) (PCL) and polyethylene glycol (PEG).

\section{a. NIPAM based polymer}

$\mathrm{N}$-Isopropylacrylamide based hydrogels are the most widely investigated thermosensitive system. The homopolymer and copolymers of Poly ( $\mathrm{N}$-isopropyl acrylamide) (PNIPAM) are investigated for their capability to deliver drugs, encapsulate cells, and regenerate tissues. PNIPAM polymers are amphiphilic carrying amide groups (hydrophilic) and isopropyl groups (hydrophobic) with the LCST at around $32{ }^{\circ} \mathrm{C}$. The LCST of the PNIPAM can be modified by co-polymerizing it with hydrophilic/hydrophobic monomers. Co-polymerization of PNIPAM with more hydrophilic monomers raises the LCST, whereas with more hydrophobic monomers, the LCST tends to decrease. The hydrophilic monomers such as acrylic acid and propyl acrylic acid modify the LCST of PNIPAM closer to body temperature. Though PNIPAM has an appropriate LCST, the clinical utilization of these thermosensitive polymers and their derivatives are limited because of their bioincompatibility [69-72].

\section{b. PEO/PPO based systems}

Poloxamers or PEO-PPO-PEO systems or Pluronics are tri-block copolymers whose thermoreversible behavior could be manipulated by varying the composition, molecular weight, and concentration at physiological temperatures. The amphiphilic nature of this polymer is due to the presence of hydrophilic ethylene oxide moiety and hydrophobic propylene oxide moiety in the structure. The varying physicochemical properties of polyethylene and polypropylene make the poloxamer block to exhibit specific gel-like properties. The temperature rise induces a micelle formation, where the moderately hydrophilic PEO chain of the poloxamer linked with the water molecules forms a shell around the hydrophobic PPO chain wrapped into it as an inner core. However, the micelle formation is also dependent on the solution concentration. When the solution concentration exceeds the critical micelle concentration, the micelle is further entrapped, accumulated, and assembled due to various forces between the micelles. The gelation of poloxamer occurs with an increase in temperature, allowing them to be utilized as a thermo-sensitive in-situ gel matrix. PEO/PPO copolymers hydrogels attract more consideration due to their thermoresponsive behavior and biocompatibility. However, their use is limited clinically as they are non-biodegradable in vivo and need to dilute by the body fluid after infusion, which cannot be accomplished over the long term [27].

\section{c. PEG/PLGA based system}

Co-polymerization of polyethylene glycol (PEG) with biocompatible polyesters delivers enticing hydrogel systems. The thermosensitive behavior of the PEG/PLGA system can be modulated by altering the length of hydrophobic polyester and PEG block significantly. PLGAPEG-PLGA tri-block copolymer has become a more fascinating thermosensitive material due to its in-toxicity, biocompatibility, and biodegradability. The sol-gel transition of PLGA-PEG-PLGA tri-block polymer is majorly due to the micelle formation which is driven by hydrophobic forces [73]. The biodegradability and thermoresponsive behavior of amphiphilic PEG/PLGA block copolymers have attracted special interest in the delivery of bioactive materials.

\section{Approaches and drug delivery system}

The thermosensitive hydrogels are most widely administered via subcutaneous, transdermal and mucosal routes [74-76].

\section{Subcutaneous drug delivery}

Unlike implants, the thermosensitive injectable hydrogels can achieve localized delivery without the requirement of invasive surgical procedures for insertion/removal of the drug delivery system. These in-situ systems release the drug in a sustained manner by undergoing a sol-gel transition at a physiological temperature [77]. If the polymer concentration is above the critical gel concentration (CGC), a gel phase will appear [78]. Subcutaneous drug delivery offers several advantages such as reduced systemic toxicity, improved patient compliance, ease in administration [79, 80]. Gong et al. synthesized a novel in-situ thermosensitive composite hydrogel based on PCL-PEG-PCL copolymer and pluronic F127 for the controlled release of chemotherapeutic drugs [81]. Subcutaneous delivery of thermosensitive PEO-PPO-PEO tri-block polymers synthesized by Cohn et al. was reported to have long-term stability and enhanced mechanical strength [82]. For successful delivery of liposomes, nanoparticles, and microspheres, an in-situ thermo-sensitive hydrogel approach would be beneficial [83]. Yang et al. demonstrated that a mixed micelle gel prepared by adding a surfactant and thermosensitive polymer can improve the solubility, stability, and drug release characteristics [84]. Chen et al. found that paclitaxel-loaded hydrogel released the drug over $21 \mathrm{~d}$ in the subcutaneous tissue and successfully suppressed tumor growth in a rat model [85]. The sol-gel transition of thermo-sensitive hydrogel given through a subcutaneous route is represented in fig. 1 .

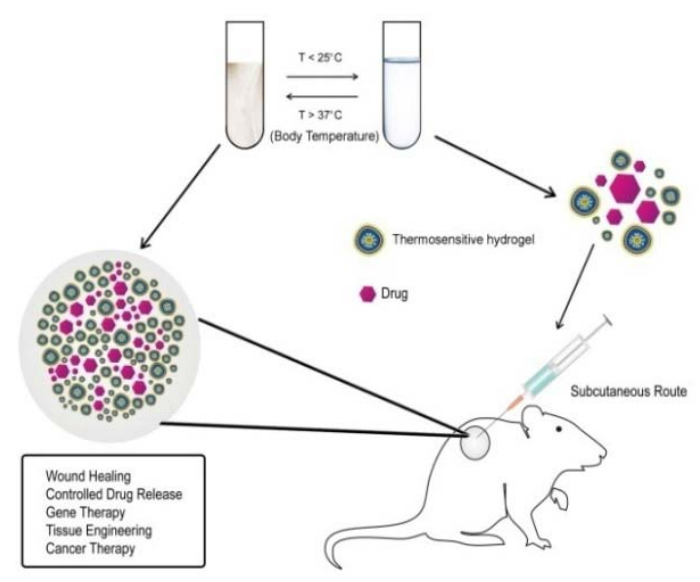

Fig. 1: This schematic diagram represents the sol-gel transition of thermo-sensitive hydrogel given through the subcutaneous route and its various applications 


\section{Transdermal drug delivery}

The transdermal route is considered to be a potential site to achieve systemic delivery of drugs. The major advantages of this route include termination of drug release at any moment is possible by simple removal of the device, controlled release of a drug over a longer duration, and avoidance of first-pass metabolism [86]. The limitation of this route is, only fewer drugs satisfy the physiochemical requirements (low dose, short half-life, low molecular weight, partition coefficient from 1.0 to 4.0 ) to permeate through the skin [87]. Gong et al., developed sustained-release insitu curcumin loaded PEG-PCL-PEG thermosensitive hydrogel to promote wound healing [88]. The thermo-sensitive sol-gel activity helps the system to adhere to wounds at skin temperature [89].

\section{Ocular drug delivery}

The ocular route is the most efficient route for topical administration of drugs that can cause systemic side effects. The ocular drug delivery system offers better bioavailability and increased drug residence time [77]. The ability to deliver liquid and semisolid dosage form via ocular route makes in-situ forming hydrogel attractive for ocular drug delivery. Cohen et al. developed a prolonged release in-situ alginate gel with higher gluconic content for ophthalmic delivery of pilocarpine, which reduces intraocular pressure over $10 \mathrm{~h}$, whereas pilocarpine solution act for the lesser duration $(3 \mathrm{~h})$ [86]. In vitro studies of oxytetracycline inserts on the rabbit eye showed a prolonged release of a drug over several days. Xi et al. reported a complete release of the drug (in 25 d) from mitomycin C loaded pluronic F 127 and poly (trimethylene carbonate) thermosensitive hydrogel [90].

\section{Rectal drug delivery}

The rectal route is considered to be the most important route for the drugs which undergo extensive first-pass metabolism. Though the administered dosage form offers considerable therapeutic efficiency in this route, the patient acceptability is poor due to the discomfort associated with the administration. The preferred dosage form for rectal administration is conventional suppositories, which are solid at room temperature and melts/softens at body temperature. The controlled release of the drug, retention of the dosage form in a specific site of the rectum, migration of dosage form upward to the colon could not be achieved with the conventional suppositories [86]. Ryu et al. reported that the bioavailability of the propranolol is increased in rats, following the incorporation of thermo-sensitive, mucoadhesive polymers like poloxamer into the conventional suppositories. Similarly, incorporation of other mucoadhesive polymers such as polycarbophil and sodium alginate into suppositories increased the bioavailability of propranolol to $82.3 \%$ and $84.7 \%$, respectively, with the highest mucoadhesive property and negligible intrarectal movement [91]. Xyloglucan, a thermosensitive gel having an intrinsic mucoadhesive property is being investigated over the past two decades for rectal administration of drugs. Xyloglucan transforms into a gel at physiological temperature. Studies showed that indomethacin-loaded xyloglucan gel administered in rabbits via rectal route have well-controlled in vivo plasma concentration and time profiles when compared to the commercial suppositories containing indomethacin without compromising the bioavailability [92].

\section{Sublingual drug delivery}

The sublingual route is the most promising route for the drugs which undergo extensive degradation by the gastrointestinal enzymes. In this route, the dosage form is kept beneath the tongue to deliver the drug directly into the blood vessels, thus helps in achieving high bioavailability by overcoming the first-pass metabolism [93]. The major limitations associated with this route are shorter mean residence time, smaller area for absorption, inadvertent swallowing of dosage form, and oral mucosal irritation [94]. The polymers like chitosan/dextran cross-linked with other polymers to obtain a thermosensitive hydrogel suitable for sublingual formulations [95]. Among the various polymers, PNIPAM has been extensively used in the preparation of thermo-responsive chitosan/dextran-based cross-linked copolymers for sublingual delivery [96]. Almeida et al. demonstrated the effect of temperature on drug release from thermo-responsive Ondansetron ${ }^{\mathrm{TM}}$ sublingual films. It was reported that the Ondansetron release is retarded at 37 ${ }^{\circ} \mathrm{C}$ compared to its release at room temperature due to the increased degree of gel cross-linking [97].

\section{Buccal drug delivery}

The buccal mucosa is an attractive target for the administration of the majority of pharmaceuticals [98]. The buccal mucosa is highly vascularized, thus allowing the drug to enter directly into the systemic circulation. The key benefits of buccal drug delivery include patient acceptability, cost-effectiveness, avoidance of gastrointestinal degradation, bypassing first-pass metabolism, rapid onset of action, and increased bioavailability [99]. Poloxamer, a thermosensitive hydrogel, has gained a special interest in buccal drug delivery owing to their sol-gel transition behavior with the response to temperature. Sandri et al. developed an in-situ thermo-sensitive buccal spray composed of poloxamer 407 (PF 127) and sodium alginate for the delivery of platelet lysate towards the treatment of oral mucositis. The poloxamer/sodium alginate spray is fluid at room temperature and rapidly forms a gel at $34^{\circ} \mathrm{C}-35^{\circ} \mathrm{C}[100]$. The poloxamer 407 (PF127)/polyethylene oxide (PEO) composite hydrogel showed a prolonged release of the drug. The rate at which the drug is released from the PF127/PEO system was highly dependent on the concentration of PEO. The increase in the PEO concentration retarded the drug release and the steady-state concentration is achieved [101,102].

\section{Applications involved in thermosensitive hydrogel}

The thermosensitive hydrogel is a very good candidate for many biomedical applications owing to their biocompatibility and their close resemblance with the extracellular matrix. Some of the typical applications of thermo-sensitive hydrogels include tissue engineering, wound healing, soft contact lenses, and sensors. Hydrogels are widely used for complex applications such as controlled drug delivery and tissue engineering rather than simple contact lenses. Some prominent examples of thermo-sensitive hydrogels in cancer therapy, protein delivery, gene therapy, tissue regeneration, and other therapeutic areas $[27,103]$ are discussed as follows.

\section{Cancer therapy}

Traditional chemotherapeutic drugs are cytotoxic and cause systemic toxicity more often. Localized delivery of chemotherapeutic drugs in a controlled manner offers better targeting and a longer duration of action, considered to be a potential approach for the treatment of cancer. Thermosensitive hydrogels as a drug delivery agent can allow the localized administration of a chemotherapeutic drug, thereby reducing their systemic side effects and increasing their efficacy. Thermosensitive hydrogels also offer controlled release of chemotherapeutic drugs at the tumor site $[104,105]$. The use of thermosensitive hydrogels in humans for cancer therapy is still under investigation. The major hindrance in thermosensitive hydrogel-based cancer therapy, especially with pluronic polymers, is the dramatic change in the tumor cellular response to sensitizing the multi-drug resistance [105]. Cho et al. used thermosensitive poly (organophosphazene) as a vehicle for the controlled delivery of angiogenic inhibitor 2-methoxy-estradiol to limit the oxygen and nutrient supply to the tumor cell to suppress the tumor growth [106]. Chitosan-based thermosensitive hydrogels are extensively used to deliver the chemotherapeutic and immuno-therapeutic agents for cancer treatment. Han et al. developed intra tumoral Doxorubicin and Vaccinia virus vaccine (Sig/E7/lAMP-1) loaded thermosensitive chitosan-based hydrogel for the treatment of tumor. This combination neither decreased and nor increased the tumor-specific CD8+T cells up to $60 \mathrm{~d}$. The survival rate of the tumor-bearing mice was significantly increased [107].

\section{Tissue regeneration}

Tissue engineering has gained special attention in the field of biomedical research due to its extensive application such as regeneration of functional tissues and delivery of bioactive components including drugs and growth factors. Thermosensitive hydrogels are found to be a promising candidate for the delivery of bioactive molecules and stem/progenitor cells needed for efficient tissue regeneration [108]. A thermosensitive hydrogel that is 
capable of delivering growth factors and cells was developed by Guan et al. Angiogenesis is the most important requirement for tissue regeneration as it can ensure the nutrient/oxygen supply. Thermosensitive hydrogels are considered to be an effective delivery vehicle for angiogenic growth factors to stimulate angiogenesis in engineered tissues [109].

\section{Gene therapy and protein delivery}

Proteins have gained special attention due to their excellent activity. However, they are more susceptible to environmental changes such as enzymes, $\mathrm{pH}$, and temperature. Thermosensitive hydrogels are widely investigated for the delivery of proteins and growth factors due to their ability to control, sustain, and achieve optimal doses at local sites for effective tissue regeneration and repair. Thermosensitive hydrogels, a carrier for the protein delivery has several advantages such as uniform dispersion of protein in three-dimensional networks to prevent aggregation, precipitation, and inactivation, avoidance of denaturation of proteins during preparation, storage, and delivery by forming a protective layer to isolate it from environment, and controlled/sustained release of proteins [110]. Chen et al. developed vascular endothelial growth factor (VEGF) loaded injectable thermosensitive poly (D, L-lactic-co-glycolic acid)-b methoxy poly (ethylene glycol) (PLGA-MPEG) hydrogel for inducing neovascularization and bone regeneration [111].

\section{Other application of thermosensitive hydrogels}

Thermosensitive hydrogels have enormous potential in several applications including wound care, cosmetology, etc. Intranasal delivery of thermosensitive hydrogels offers efficient systemic drug delivery and has the potential to bypass the blood-brain barrier by altering the permeability in the nasal cavity $[112,113]$. Thermosensitive hydrogel allows heat dissipation, mimicking the biological sweating phenomenon [114]. Thermosensitive hydrogels are excellent carriers for the delivery of various biotherapeutic molecules as it can protect the incorporated cells and release them in a controlled manner [27].

Table 1: Summary of various thermosensitive hydrogels

\begin{tabular}{|c|c|c|c|c|c|}
\hline Methods & Polymers & Drugs & Formulation & Treatment & Reference \\
\hline Thin-layer evaporation & Chitosan, Poloxamer & Opiorphin & Liposomes & Liver cancer & [115] \\
\hline Ammonium sulfate sodium method & Carbopol, HPMC & Doxorubicin & Liposomes & Liver cirrhosis & [116] \\
\hline Emulsion evaporation method & Chitosan, gelatin & Curcumin & Nanoparticle & glaucoma & [117] \\
\hline Melt emulsification method & MPEG-PCL & Docetaxel & Nanoparticle & Anti-ovarian cancer & [118] \\
\hline $\begin{array}{l}\text { Emulsification and solvent } \\
\text { diffusion method }\end{array}$ & HPMC, Pluronic-F 127 & Sertoconazole & $\begin{array}{l}\text { Nanostructured } \\
\text { lipid carrier }\end{array}$ & Fungal keratitis & [119] \\
\hline Hot emulsification method & Chitosan & Methotrexate & Nanotubes & Control tumor cell growth & {$[120]$} \\
\hline Ethanol injection method & $\begin{array}{l}\text { Poloxamer 407, HPMC } \\
\text { K100, Carbopol } 934\end{array}$ & Zolmitriptan & Nanoethosomes & Treat headache disorders & {$[121]$} \\
\hline Simple thin-film method & $\begin{array}{l}\text { Pluronic F-127 } \\
\text { andPluronic L } 121\end{array}$ & $\begin{array}{l}\text { Doxorubicin } \\
\text { and Docetaxel }\end{array}$ & Micelles & Treat tumor & {$[122]$} \\
\hline $\begin{array}{l}\text { Rotary evaporation sonication } \\
\text { technique }\end{array}$ & Pluronic F-127 & Insulin & Transfersomes & Treat diabetes mellitus & [123] \\
\hline Emulsion cross-linking method & Chitosan, PF-127 & Lorazepam & Microsphere & Treat epilepsy & {$[124]$} \\
\hline Fluorescence imaging method & $\begin{array}{l}\text { Amphiphilic } \\
\text { copolymer }\end{array}$ & Paclitaxel & Free drug & $\begin{array}{l}\text { Intraperitoneal chemotherapy } \\
\text { of carcinomatosis }\end{array}$ & {$[125]$} \\
\hline Wang's method & $\begin{array}{l}\text { Hydroxy-butyl } \\
\text { chitosan }\end{array}$ & Dopamine & Free drug & Hemostasis & {$[126]$} \\
\hline
\end{tabular}

\section{CONCLUSION}

Thermo-sensitive hydrogels based on natural and synthetic polymers exhibit lower critical solution temperature, low inflammatory response, biocompatibility, biodegradability, and mechanical properties suitable for various applications such as cancer therapy, protein delivery, gene delivery, tissue regeneration, and wound care. The route of administration and physiochemical properties of the drug has an important effect on the selection of thermo-sensitive polymers. Commercially available and FDA approved PF-127 is the most used thermo-sensitive polymer to date. Evidence from various in vitro and in vivo studies showed that existing thermo-sensitive hydrogels exhibits reduced cell viability and reduced capability to deliver the cells to the target tissues. However, with the appropriate experimentation, these limitations could be easily addressed. On the other hand, the properties of thermo-sensitive hydrogel should be improved to enable its usage in clinical practice.

\section{FUNDING}

Nil

\section{AUTHORS CONTRIBUTIONS}

All the authors have contributed equally.

\section{CONFLICT OF INTERESTS}

Declared none

\section{REFERENCES}

1. Xing JF, Zheng ML, Duan XM. Two-photon polymerization microfabrication of hydrogels: an advanced 3D printing technology for tissue engineering and drug delivery. Chem Soc Rev 2015;44:5031-9.

2. Macaya D, Spector M. Injectable hydrogel material for spinal cord regeneration: a review. Biomed Mater 2012;7:012001.

3. Surojeet D, Vivek K, Rini T, Leena S, Sachidanand S. Recent advances in hydrogels for biomedical applications. Asian J Pharm Clin Res 2018;11:62-8.

4. Sabbagh F, Kiarostami K, Khatir NM, Rezania S, Muhamad I. Green synthesis of $\mathrm{Mg} 0.99 \mathrm{Zn} 0.010$ nanoparticles for the fabrication of $\kappa$-carrageenan/NaCMC hydrogel to deliver catechin. Polym 2020;12:861.

5. Hu W, Wang Z, Xiao Y, Zhanga S, Wang J. Advances in crosslinking strategies of biomedical hydrogels. Biomater Sci 2019;7:843-55.

6. Sabbagh F, Khatir NM, Karim AK, Omidvar A, Nazari Z, Jaberi R Mechanical properties and swelling behavior of acrylamide hydrogels using montmorillonite and kaolinite as clays. J Environ Treat Tech 2011;7:211-9.

7. Huh HW, Zhao L, Kim SY. Biomineralized biomimetic organic/inorganic hybrid hydrogels on hyaluronic acid and poloxamer. Carbohydr Polym 2015;1:130-40.

8. Garima S, Alka L, Shiv Sankar B. Hydrogel as a novel drug delivery system: a review. J Fundam Pharm Res 2014;2:35-48.

9. Tibbitt MW, Anseth KS. Hydrogels as extracellular matrix mimic for 3D cell culture. Biotechnol Bioeng 2009;103:655-63.

10. Jamstorp Berg E. Diffusion controlled drug release from slurry formed, porous, organic, and clay-derived pellets (Doctoral dissertation. Acta Universitatis Upsaliensis; 2012. p. 80.

11. Nesrinne S, Djamel A. Synthesis, characterization, and rheological behavior of pH-sensitive poly (acrylamide-coacrylic acid) hydrogels. Arabian J Chem 2017;10:539-47. 
12. Anita D, Ujjwal N, Sarabjot K, Komal. Hydrogels: a smart drug delivery device. Asian Pac J Health Sci 2014;1:92-105.

13. Matanovic MR, Kristl J, Grabnar PA. Thermoresponsive polymers: Insights into decisive hydrogel characteristics, mechanisms of gelation, and promising biomedical applications. Int J Pharm 2014;472:262-75.

14. Ishida $\mathrm{K}$, Uno $\mathrm{T}$, Itoh $\mathrm{T}$, Kubo M. Synthesis and property of temperature-responsive hydrogel with movable cross-linking points. Macromolecules 2012;45:6136-42.

15. Liu YY, Shao YH, Jian L. Preparation, properties and controlled release behaviors of $\mathrm{pH}$-induced thermosensitive amphiphilic gels. Biomaterials 2006;27:4016-24.

16. Qiu Y, Park K. Environment-sensitive hydrogels for drug delivery. Adv Drug Delivery Rev 2001;53:321-39.

17. Elena B, Andrea LS, Steven RL, Paolo D. Injectable thermoresponsive hydrogels as a drug delivery system for the treatment of central nervous system disorders: a review. J Controlled Release 2021;329:19-9.

18. Gariepy ER, Leroux JC. In situ-forming hydrogels-review of temperature-sensitive systems. Eur J Pharm Biopharm 2004;58:409-26.

19. Fundueanu G, Constantin M, Asmarandei I, Bucatariu S, Harabagiu V, Scenzi P, et al. Poly (N-isopropyl acrylamide-cohydroxy ethyl acrylamide) thermosensitive microspheres: the size of microgels dictates the pulsatile release mechanism. Eur J Pharm Biopharm 2013;85:614-23.

20. Lee JW, Jung MC, Park HD, Park KD, Ryu GH. Synthesis and characterization of thermosensitive chitosan copolymer as a novel biomaterial. J Biomater Sci Polym Ed 2004;15:1065-79.

21. Ward MA, Georgiou TK. Thermoresponsive terpolymers based on methacrylate monomers: effect of architecture and composition. J Polym Sci Part A: Polym Chem 2010;48:775-83.

22. Li Z, Wang F, Roy S, Sen CK, Guan J. Injectable, highly flexible, and thermosensitive hydrogels capable of delivering superoxide dismutase. Biomacromolecules 2009;10:3306-16.

23. Chitra G, Vijay J, Upendra N. Formulation and optimization of thermosensitive in-situ gel of moxifloxacin hydrochloride for ocular drug delivery. Int J Appl Pharm 2018;3:123-30.

24. Gong C, Qi T, Wei X, Qu Y, Wu Q, Luo F, et al. Thermosensitive polymeric hydrogels as drug delivery systems. Curr Med Chem 2013;20:79-94.

25. Li SK, Emanuele AD. On-off transport through a thermoresponsive hydrogel composite membrane. J Controlled Release 2001;75:55-67.

26. Schild HG, Tirrell DA. Micro calorimetric detection of lower critical solution temperatures in aqueous polymer solutions. J Phys Chem 1990;94:4352-6.

27. Huang $\mathrm{H}$, Qi X, Chen $\mathrm{Y}, \mathrm{Wu} \mathrm{Z}$. Thermo-sensitive hydrogels for delivering biotherapeutic molecules: a review. Saudi Pharm J 2019;27:990-9.

28. Sudipta C, Patrick CH, Kan C. Thermoresponsive hydrogels and their biomedical applications: special insight into their applications in textile based transdermal therapy. MDPI 2018:10:480

29. Pillai O, Panchagnula R. Polymers in drug delivery. Curr Opin Chem Biol 2001;5:447-51.

30. Li L, Shan H, Yue CY, Lam YC, Tam KC, Hu X. Thermally induced association and dissociation of methylcellulose in aqueous solutions. Langmuir 2002;18:7291-8.

31. Takahashi M, Shimazaki M, Yamamoto J. Thermoreversible gelation, and phase separation in aqueous methylcellulose solutions. J Polym Sci Part B: Polym Phys 2001;39:91-100.

32. Liu $\mathrm{W}$, Zhang B, Lu WW, Li X, Zhu D, De Yao K, et al. A rapid temperature-responsive sol-gel reversible poly ( $\mathrm{N}$-isopropyl acrylamide)-g-methylcellulose copolymer hydrogel. Biomaterials 2004;25:3005-12

33. Maite AP, Leyre PA, Luis Carlos CI, Issa K. Biodegradable chitosan nano gels crosslinked with genipin. Carbohydr Polym 2013;94:836-42.

34. Xiao C, You R, Fan Y, Zhang Y. Tunable functional hydrogels formed from versatile water-soluble chitosan. Int J Biol Macromol 2016;85:386-90

35. Hamidi M, Azadi A, Rafiei P. Hydrogel nanoparticles in drug delivery. Adv Drug Delivery Rev 2008;60:1638-49.
36. Esquerdo V, Cadaval T, Dotto G, Pinto L. Chitosan scaffold as an alternative adsorbent for the removal of hazardous food dyes from aqueous solutions. J Colloid Interface Sci 2014;424:7-15.

37. Chenite A, Chaput C, Wang D, Combes C, Buschmann MD, Hoemann CD, et al. Novel injectable neutral solutions of chitosan form biodegradable gels in situ. Biomaterials 2000;21:2155-61.

38. Qi FD, Jing QY, Hong L, Cheng SL, Xi GC, Qiu XJ, et al. Biological evaluation of chitosan-based in-situ forming hydrogel with a low phase transition temperature. J Appl Polym Sci 2015;132:41594.

39. Anuja N, Hema N. Formulation and evaluation of thermosensitive bio gels for the nose to brain delivery of doxepin. Biomed Res Int 2014 https://doi.org/10.1155/2014/847547

40. Supper S, Anton N, Seidel N, Riemenschnitter M, Schoch C, Vandamme T. Rheological study of chitosan/polyol-phosphate systems: influence of the polyol part on the thermo-induced gelation mechanism. Langmuir 2013;29:10229-37.

41. Bhattarai N, Matsen FA, Zhang M. PEG-grafted chitosan as an injectable thermoreversible hydrogel. Macromol Biosci 2005;5:107-11.

42. Nazara H, Fatouros DG, Van der Merwe SM, Bouropoulos G, Avgouropoulos G, Tsibouklis J, et al. Thermosensitive hydrogels for nasal drug delivery: The formulation and characterization of systems based on N-trimethyl chitosan chloride. Eur J Pharm Biopharm 2011;77:225-32.

43. Semenzato A, Costantini A, Baratto G. Green polymers in personal care products: rheological properties of tamarind seed polysaccharide. Cosmetics 2015;2:1-10.

44. Martinez Ibarra DM, Lopez Cervantes J, Sanchez Machado DI Sanches Silva A. Chitosan and xyloglucan-based hydrogels: an overview of synthetic and functional utility. chitin-chitosanmyriad functionalities in science and technology. Intech Open 2018;184-18:74646.

45. Pique N, Carmen Gomez Guillen MD, Montero MP. Xyloglucan, a plant polymer with barrier protective properties over the mucous membranes: an overview. Int J Mol Sci 2018;19:673

46. Picone P, Sabatino MA, Ajovalasit A, Giacomazza D, Dispenza C, Di Carlo M. Biocompatibility, hemocompatibility and antimicrobial properties of xyloglucan based hydrogel film for wound healing application. Int J Biol Macromol 2019;121:784-95.

47. Zhang E, Li L, Zhou Y, Che P, Ren B, Qin Z, et al. Biodegradable and injectable thermoreversible xyloglucan-based hydrogel for prevention of postoperative adhesion. Acta Biomater 2017:55:420-33.

48. Amanda K, Brun Graeppi AS, Richard C, Bessodes M, Scherman D, Narita T, et al. Study on the sol-gel transition of xyloglucan hydrogels. Carbohydr Polym 2010;80:555-63.

49. Moneo Sanchez M, Alonso Chico A, Knox JP, Dopico B, Labrador E, Martin I. $\beta-(1,4)$-galactan remodeling in arabidopsis cell walls affects the xyloglucan structure during elongation. Planta 2018;249:351-62.

50. Chen D, Guo P, Chen S, Cao Y, Ji W, Lei X, et al. Properties of xyloglucan hydrogel as the biomedical sustained-release carriers. J Mater Sci Mater Med 2012;23:955-62.

51. Ajovalasit A, Sabatino MA, Todaro S, Alessi S, Giacomazza D, Picone $\mathrm{P}$, et al. Xyloglucan-based hydrogel films for wound dressing: structure-property relationships. Carbohydr Polym 2017:179:262-72.

52. Mahajan HS, Tyagi V, Lohiya G, Nerkar P. Thermally reversible xyloglucan gels as vehicles for nasal drug delivery. Drug Delivery 2012;19:270-6.

53. Kulkarni AD, Joshi AA, Patil CL, Amale PD, Patel HM, Surana SJ, et al. Xyloglucan: a functional biomacromolecule for drug delivery applications. Int J Biol Macromol 2017;104:799-12.

54. Simi CK, Abraham TE. Transparent xyloglucan-chitosan complex hydrogels for different applications. Food Hydrocoll 2010;24:72-80

55. Nisbet DR, Rodda AE, Horne MK, Forsythe JS, Finkelstein DI. Implantation of functionalized thermally gelling xyloglucan hydrogel within the brain: associated neurite infiltration and inflammatory response. Tissue Eng Part A 2010;16:2833-42.

56. Ye L, Wu X, Mu Q, Chen B, Duan YH, Geng X, et al. Heparinconjugated PCL scaffolds fabricated by electrospinning and 
loaded with fibroblast growth factor 2. J Biomater Sci Polym Ed 2011;22:389-406.

57. Silva AK, Richard C, Ducouret G, Bessodes M, Scherman D, Merten OW. Xyloglucan-derivatized films for the culture of adherent cells and their Thermo controlled detachment: a promising alternative to cells sensitive to protease treatment. Biomacromolecules 2013;14:512-9.

58. Prabaharan M, Mano JF. Stimuli-responsive hydrogels based on polysaccharides incorporated with thermo-responsive polymers as novel biomaterials. Macromol Biosci 2006;6:991-1008.

59. Kim MS, Park SJ, Chun HJ, Kim CH. Thermosensitive hydrogels for tissue engineering. Tissue Eng Regener Med 2011;8:117-23.

60. Young S, Wong M, Tabata Y, Mikos AG. Gelatin as a delivery vehicle for the controlled release of bioactive molecules. J Controlled Release 2005;109:256-74.

61. Joly Duhamel C, Hellio D, Djabourov M. All gelatin networks: 1 . Biodiversity and physical chemistry. Langmuir 2002;18:7208-17.

62. Das N. Bio-degradable hydrogels for controlled drug delivery. Cellulose Based Super Absorbent Hydrogels 2019;48:1434-70.

63. Yang $\mathrm{H}, \mathrm{Kao} \mathrm{WJ}$. Thermoresponsive gelatin/mono methoxy poly (ethylene glycol)-poly (d, l-lactide) hydrogels: formulation, characterization, and antibacterial drug delivery. Pharm Res 2006;23:205-14.

64. Alexander A, Ajazuddin, Khan J, Saraf S, Saraf S. Poly (ethylene glycol)-poly (lactic-co-glycolic acid) based thermosensitive injectable hydrogels for biomedical applications. J Controlled Release 2013;172:715-29.

65. Leda K. Thermoresponsive hydrogels in biomedical applications: a seven-year update. Eur J Pharm Biopharm 2015;97:338-49.

66. Wang Q, Zuo Z, Chucky Cheung CK, Yee Leung SS. Updates on thermosensitive hydrogel for nasal, ocular and cutaneous delivery. Int J Pharm 2019;559:86-101.

67. Le PN, Huynh CK, Tran NQ. Advances in thermosensitive polymer-grafted platforms for biomedical applications. Mater Sci Eng C Mater Biol Appl 2018;92:1016-30.

68. Darge HF, Andrgie AT, Tsai HC, Lai JY. Polysaccharide and polypeptide based injectable thermosensitive hydrogels for local biomedical applications. Int J Biol Macromol 2019;133:545-63.

69. Yin X, Hoffman AS, Stayton PS. Poly (N-isopropyl acrylamideco-propyl acrylic acid) copolymers that respond sharply to temperature and $\mathrm{pH}$. Biomacromolecules 2006;7:1381-5.

70. Masamichi N, Teruo O, Takanari M, Fukashi K, Kiyotaka S, Masayuki Y. Molecular design of biodegradable polymeric micelles for temperature-responsive drug release. J Controlled Release 2006;115:46-56.

71. Coughlan DC, Quilty FP, Corrigan OI. Effect of drug physicochemical properties on swelling/deswelling kinetics and pulsatile drug release from thermoresponsive poly $(\mathrm{N}-$ isopropyl acrylamide) hydrogels. J Controlled Release 2004;98:97-114.

72. Pei Y, Chen J, Yang L, Shi L, Tao Q, Hui B, et al. The effect of $\mathrm{pH}$ on the LCST of poly (N-isopropyl acrylamide) and poly $(\mathrm{N}$ isopropyl acrylamide-co-acrylic acid). J Biomater Sci Polym Ed 2004;15:585-94.

73. Kim YJ, Kim SW. Controlled drug delivery from the injectable biodegradable triblock copolymer. ACS Sympos 2002;833:300-11.

74. Tirtaatmadja N, Murphy KT, Lynch GS, Connor OA. Mixed micelles to deliver drugs for skeletal muscle regeneration. In: Proceedings of the international society, Asia Pacific Meeting, Sydney, Australia, September; 2010.

75. Gulsen D, Chauhan A. Dispersion of microemulsion drops in HEMA hydrogel: a potential ophthalmic drug delivery vehicle. Int J Pharm 2005;292:95-117.

76. Kim J, Conway A, Chauhan A. Extended delivery of ophthalmic drugs by silicone hydrogel contact lenses. Biomaterials 2008;29:2259-69.

77. Lin Z, Gao W, Hu H, Ma K, He B, Dai W, et al. Novel thermosensitive hydrogel system with paclitaxel nanocrystals: high drug-loading, sustained drug release and extended local retention guaranteeing better féfacy and lower toxicity. J Controlled Release 2014;174:161-70.

78. Jeong B, Kim SW, Bae YH. Thermosensitive sol-gel reversible hydrogels. Adv Drug Delivery Rev 2002;54:37-51.
79. Madan M, Bajaj A, Lewis S, Udupa N, Baig J. In situ forming polymeric drug delivery systems. Indian J Pharm Sci 2009;71:242-51.

80. Lin Z, Mei D, Chen M, Wang Y, Chen X, Wang Z, et al. A comparative study of thermo-sensitive hydrogels with waterinsoluble paclitaxel in the molecule, nanocrystal, and microcrystal dispersions. Nanoscale 2015;7:14838-47.

81. Gong CY, Shi S, Dong PW, Zheng XL, Fu SZ, Guo G, et al. In vitro drug release behavior from a novel thermosensitive composite hydrogel based on pluronic f127 and poly (ethylene glycol)poly ( $\varepsilon$-caprolactone)-poly (ethylene glycol) copolymer. BMC Biotechnol 2009;9:8.

82. Cohn D, Sosnik A, Malal R, Zarka R, Garty S, Levy A. Chain extension as a strategy for the development of improved reverse thermoresponsive polymers. Polym Adv Technol 2007;18:731-6.

83. Yang Z, Nie S, Hsiao WW, Pam W. Thermo reversible Pluronic ${ }^{\circledR}$ F127-based hydrogel containing liposomes for the controlled delivery of paclitaxel: In vitro drug release, cell cytotoxicity, and uptake studies. Int J Nanomed 2011;6:151-66.

84. Yang Y, Wang J, Zhang X, Lu W, Zhang Q. A novel mixed micelle gel with thermo-sensitive property for the local delivery of docetaxel. J Controlled Release 2009;135:175-82.

85. Cheng Y, He C, Ding J, Xiao C, Zhuang X, Chen X. Thermo-sensitive hydrogels based on polypeptides for localized and sustained delivery of anticancer drugs. Biomaterials 2013;34:10338-47.

86. Bindu Sri M, Ashok V, Chatterjee A. A review on hydrogels as drug delivery in the pharmaceutical field article. Int J Pharm Chem Sci 2012;1:642-61.

87. Ali S, Yosipovitch G, Skin PH. From basic science to basic skin care. Acta Derm Venereol 2013;93:261-7.

88. Gong $\mathrm{C}, \mathrm{Wu} \mathrm{Q}$, Wang $\mathrm{Y}$, Zhang $\mathrm{D}$, Luo $\mathrm{F}$, Zhao $\mathrm{X}$, et al. $\mathrm{A}$ biodegradable hydrogel system containing curcumin encapsulated in micelles for cutaneous wound healing. Biomaterials 2013;34:6377-87.

89. Chen $\mathrm{X}$, Peng LH, Shan YH, Li N, Wei W, Yu L, et al. Astragaloside IV-loaded nanoparticle-enriched hydrogel induces wound healing and anti-scar activity through topical delivery. Int J Pharm 2013;447:171-81.

90. Xi L, Wang T, Zhao F, Zheng Q, Li X, Luo J, et al. Evaluation of an Injectable thermosensitive hydrogel as drug delivery implant for ocular glaucoma surgery. PLoS One 2014;9:e100632.

91. Ryu JM, Chung SJ, Lee MH, Kim CK, Shim CK. Increased bioavailability of propranolol in rats by retaining thermally gelling liquid suppositories in the rectum. J Controlled Release 1999:59:163-72.

92. Miyazaki S, Suisha F, Kawasaki N, Shirakawa M, Yamatoya K, Attwood D. Thermally reversible xyloglucan gels as vehicles for rectal drug delivery. J Controlled Release 1998;56:75-83.

93. Goswami T, Jasti B, Li X. Sublingual drug delivery. Crit Rev Ther Drug Carrier Syst 2008;25:449-84.

94. Wang Z, Chow MS. Overview and appraisal of the current concept and technologies for improvement of sublingual drug delivery. Ther Delivery 2014;5:807-16.

95. Ferreira L, Gil MH, Dordick JS. Enzymatic synthesis of dextrancontaining hydrogels. Biomaterials 2002;23:3957-67.

96. Salgado Rodriguez R, Licea Claverie A, Arndt KF. Random copolymers of $\mathrm{N}$ Isopropyl acrylamide and methacrylic acid monomers with hydrophobic spacers: $\mathrm{pH}$ tunable temperaturesensitive materials. Eur Polym J 2004;40:1931-46.

97. Almeida JF, Ferreira P, Alves P, Lopes A, Gil MH. Thermalresponsive hydrogels for sublingual administration of Ondansetron $^{\text {TM }}$. Int $\mathrm{J}$ Polymeric Materials Polymeric Biomaterials 2017;67:765-75.

98. Chinna Reddy P, Chaitanya KSC, Madhusudan Rao Y. A review on bioadhesive buccal drug delivery systems: current status of formulation and evaluation methods. Daru 2011;19:385-403.

99. Zenga N, Dumortier G, Maury M, Mignet N, Boudya V. Influence of additives on a thermosensitive hydrogel for buccal delivery of salbutamol: relation between micellization, gelation, mechanic and release properties. Int J Pharm 2014;467:70-83.

100. Sandri G, Bonferroni MC, Ferrari F, Rossi S, Del Fante C, Perotti $\mathrm{C}$, et al. An in situ gelling buccal spray containing platelet lysate for the treatment of oral mucositis. Curr Drug Discovery Technol 2011;8:277-85. 
101. Park JS, Oh YK, Yoon H, Kim JM, Kim CK. In situ gelling and mucoadhesive polymer vehicles for controlled intranasal delivery of plasmid DNA. J Biomed Mater Res 2002;59:144-51.

102. Choi SG, Lee SE, Kang BS, Ng CL, Davaa E. Thermosensitive and mucoadhesive sol-gel composites of paclitaxel/dimethyl-bcyclodextrin for buccal delivery. PLoS One 2014;9:e109090.

103. Vermonden T, Fedorovich NE, Geemen DV, Alblas J, van Nostrum CF. Photo-polymerized thermosensitive hydrogels: synthesis, degradation, and cytocompatibility. Biomacromolecules 2008;9:919-26.

104. Kabanov AV. Polymer genomics: an insight into pharmacology and toxicology of nanomedicines. Adv Drug Delivery Rev 2006;58:1597-621.

105. Turabee MH, Jeong TH, Ramalingam P, Kang JH, Ko YT. N, N, Ntrimethyl chitosan embedded in situ pluronic F127 hydrogels for the treatment of brain tumor. Carbohydr Polym 2019;203:302-9.

106. Cho JK, Hong KY, Park JW, Yang HK, Song SC. The injectable delivery system of 2-methoxy estradiol for breast cancer therapy using biodegradable thermosensitive poly (organophosphazene) hydrogel. J Drug Target 2011;19:270-80.

107. Han HD, Song CK, Park YS, Noh KH, Kim JH, Hwang T, et al. A chitosan hydrogel-based cancer drug delivery system exhibits synergistic antitumor effects by combining with a vaccinia viral vaccine. Int J Pharm 2008;350:27-34.

108. Kwon JS, Park IK, Cho AS, Shin SM, Hong MH, Jeong SY, et al. Enhanced angiogenesis mediated by vascular endothelial growth factor plasmid-loaded thermo-responsive amphiphilic polymer in a rat myocardial infarction model. J Controlled Release 2009;138:168-76.

109. Oh KS, Song JY, Yoon SJ, Park Y, Kim D, Yuk SH. Temperatureinduced gel formation of core/shell nanoparticles for the regeneration of the ischemic heart. J Controlled Release 2010;146:207-11

110. Xu HL, Xu J, Zhang SS, Zhu QY, Jin BH, Zhuge DL, et al. Temperature-sensitive heparin-modified poloxamer hydrogel with affinity to KGF facilitate the morphologic and functional recovery of the injured rat uterus. Drug Delivery 2017;24:86781.

111. Chen D, Zhang C, Huo H, Ji C, Sun M, Nie L. Injectable temperature-sensitive hydrogel with VEGF loaded microspheres for vascularization and bone regeneration of femoral head necrosis. Mater Lett 2018;229:138-41.

112. Wong YC, Zuo Z. Brain disposition and catalepsy after intranasal delivery of loxapine: role of metabolism in PK/PD of intranasal CNS drugs. Pharm Res 2013;30:2368-84.

113. Deshpande ST, Lahoti SR, Dhamecha DL, Rajendra VB, Dehghan MHG, Puranik PK. Enhanced iontophoretic delivery of sumatriptan succinate from the thermosensitive gel using chemical enhancers. Indian J Pharm Ed Res 2013;47:27-33.

114. Shirui Pu, Jiaxuan Su, Liuxiang Li, Wang Hs, Chen C, Xuejiao Hu. Bioinspired sweating with temperature-sensitive hydrogel to passively dissipate heat from high-end wearable electronics. Energy Conversion Management 2019;180:747-56.

115. Mura P, Mennini N, Nativi C, Richichi B. In situ mucoadhesivethermosensitive liposomal gel as a novel vehicle for nasal extended delivery of opiorphin. Eur J Pharm Biopharm 2018;122:54-61.

116. Shuangxia R, Yu D, Cuiyun L, Qiu Z, Wang X, Tian F, et al. Pharmacokinetics and pharmacodynamics evaluation of a thermosensitive chitosan-based hydrogel containing liposomal doxorubicin. Eur J Pharm Sci 2016;92:137-45.

117. Cheng YH, Ko YC, Chang YF, Huang SH, Liu CJ. The thermosensitive chitosan-gelatin-based hydrogel containing curcumin-loaded nanoparticles and latanoprost as a dual-drug delivery system for glaucoma treatment. Exp Eye Res 2019;179:179-87.

118. Xu G, Zhu C, Li B, Wang T, Wan J, Zhang Y. improving the antiovarian cancer activity of docetaxel by self-assemble micelles and thermosensitive hydrogel drug delivery system. J Biomed Nanotechnol 2020;16:40-53.

119. Tavakoli N, Taymouri S, Saeidi A, Akbari V. Thermosensitive hydrogel containing sertaconazole loaded nanostructured lipid carriers for the potential treatment of fungal keratitis. Pharm Dev Technol 2019;24:891-901.

120. Saeednia L, Yao L, Cluff K, Asmatulu R. sustained releasing of methotrexate from injectable and thermosensitive chitosancarbon nanotube hybrid hydrogels effectively controls tumor cell growth. ACS Omega 2019;4:4040-8.

121. Shelke S, Shahi S, Jalalpure S, Dhamecha D. Poloxamer 407based intranasal thermoreversible gel of zolmitriptan-loaded nanoethosomes: formulation, optimization, evaluation, and permeation studies. J Liposome Res 2016;26:313-23.

122. Sheu MT, Jhan HJ, Su CY, Chen LC, Chang CE, Liu DZ, et al. Codelivery of doxorubicin containing thermosensitive hydrogels incorporated with docetaxel-loaded mixed micelles enhance local cancer therapy. Colloids Surfaces B 2016;143:260-70.

123. Marwah H, Garg T, Rath G, Goyal AK. Development of transferosomal gel for transdermal delivery of insulin using iodine complex. Drug Delivery 2016;23:1636-44.

124. Jose S, Ansa CR, Cinu TA, Chacko AJ, Aleykutty NA, Ferreira SV et al. Thermo-sensitive gels containing lorazepam microspheres for intranasal brain targeting. Int J Pharm 2013;441:516-26.

125. Xu S, Fan H, Yin L, Zhang J, Dong A, Deng LD, et al. Thermosensitive hydrogel system assembled by PTX-loaded copolymer nanoparticles for sustained intraperitoneal chemotherapy of peritoneal carcinomatosis. Eur J Pharm Biopharm 2016;104:251-9.

126. Zhang X, Sun GH, Tian MP, Wang YN, Qu CC, Cheng XJ, et al. Mussel-inspired antibacterial polydopamine/chitosan/temperature-responsive hydrogels for rapid hemostasis. Int J Biol Macromol 2019;138:321-33. 